\section{Short-Call Abstracts}

\section{EXPOSURE TO HIGH THYROID HORMONE IN UTERO CAUSES PERMANENT REDUCED SENSITIVITY TO THYROID HORMONE Joao Anselmo ${ }^{1}$, Alexandra M. Dumitrescu², Roy E. Weiss ${ }^{3}$, Samuel Refetoff \\ ${ }^{1}$ Serviço de Endocrinologia e Nutrição, Hospital Divino Espírito Santo, Ponta Delgada, Azores-Portugal; ${ }^{2}$ Department of Medicine, The University of Chicago, Chicago, IL; ${ }^{3}$ Department of Medicine, The University of Miami, Miami, FL; ${ }^{4}$ Departments of Medicine, Pediatrics, and the Committee on Genetics, The University of Chicago, Chicago, IL, USA}

Objectives: High maternal thyroid hormone levels have a deleterious effect on fetal growth and development. We have shown that unaffected fetuses carried by mothers with resistance to thyroid hormone due to mutations in the THRB gene (RTH-beta) are more often aborted, and when born, have lower weight and suppressed TSH. We also showed that serum TSH in adult unaffected mice born to dams with RTH-beta is less suppressible by T3. The objective of this study is to determine if this long term effect also occurs in humans.

Methods: Normal adult individuals (without THRB gene mutations), aged 22-54 yrs born to affected mothers and to affected fathers $(n=4: 3 F$ and $1 \mathrm{M}$ in each group) and belonging to the same extended family with THRB R243Q mutation were given $200 \mu \mathrm{g}$ TRH iv before and after 3 day treatment with $25 \mu \mathrm{g} \mathrm{L}$-T3 twice daily. Serum was collected at $-15,0,15,30,45,60$, 90, 120 and $180 \mathrm{~min}$ after TRH. Analysis was done by ANOVA. Results are expressed as mean \pm SD.

Results: Basal values of serum TSH, FT4, FT3, rT3, TG, and PRL were not significantly different, and after L-T3 treatment, both groups had equally elevated serum T3 and suppressed TSH. Before L-T3 treatment, peak TSH response to TRH in individuals born to affected mothers compared to those born to affected fathers were $11.8 \pm 1.7 \mathrm{mU} / \mathrm{L}$ vs $13.2 \pm 1.7 \mathrm{mU} / \mathrm{L} ; \mathrm{P}=0.57$. Following L-T3 treatment corresponding values were $6.8 \pm 1.0$ vs $1.6 \pm 0.5$; $\mathrm{P}<0.005$. While before L-T3 treatment the TSH response to TRH in both groups overlapped, there was no overlap between the groups after L-T3 treatment. There were no differences in basal and stimulated PRL between the two groups before and after L-T3 treatment.

Conclusions: Exposure to high thyroid hormone in utero resets the sensitivity of the thyrotrophs to L-T3, producing persistent, subclinical RTH into adulthood.

\section{TRIAC TREATMENT OF AN INFANT WITH ALLAN-HERNDON-DUDLEY SYNDROME (AHDS): EFFECTS ON IODOTHYRONINES IN SERUM AND CEREBRO-SPINAL FLUID \\ Ainhoa Iglesias ${ }^{1}$, Olga Esther Alonso², Ana Gómez-Gila², \\ Juan A. Campos-Cerveró ${ }^{3}$, María Palomares ${ }^{4}$, Eduvigis Contreras ${ }^{5}$, Beatriz Morte ${ }^{6}$, Maria Jesús Obregón ${ }^{6}$, Juan Bernal ${ }^{6}$, José C. Moreno ${ }^{1}$ ${ }^{1}$ Thyroid Molecular Laboratory, INGEMM, La Paz University Hospital (HULP), Madrid, Spain, ${ }^{2}$ Pediatric Endocrinology and Neurology, Virgen del Rocío Hospital, Sevilla, ${ }^{3}$ Rehabilitation, S. Juan de Dios Hospital, Sevilla, ${ }^{4}$ INGEMM, HULP, ${ }^{5}$ Paidopsychology, HULP, ${ }^{6}$ Institute for Biomedical Research (IIB), CIBERER, CSIC, Madrid, Spain}

Allan-Herndon-Dudley Syndrome (AHDS) is a devastating disease caused by defects in the thyroid hormone $(\mathrm{TH})$ transporter MCT8. Systemic hyperthyroidism is heralded by elevated serum T3 (with mildly increased TSH and decreased T4). However, the brain is hypothyroid, causing incapacitating psychomotor retardation. Therapeutic attempts with PTU+Levothyroxine or the T3-analogue DITPA could normalize TH derangements without neurological improvement. Recently, administration of TETRAC to Mct8 KO mice corrected TH abnormalities in this model for AHDS.

Objective: To determine the TH metabolic and developmental effects of TRIAC (a rapidly-generated metabolite of TETRAC) in a child with earlydiagnosed AHDS.

Patient and Methods: 8-month-old male with severe axial hypotonia, psychomotor retardation and apparent hypothyroidism (TSH $7.7 \mathrm{mU} / \mathrm{L}$, FT4 $0.48 \mathrm{ng} / \mathrm{dL})$ but elevated FT3 $(8.86 \mathrm{pg} / \mathrm{mL})$. Thyroid-targeted CGH array (Thyroarray $\left.{ }^{\mathcal{O}}\right)$. Compassionate treatment with increasing doses of TRIAC $(10-40 \mu \mathrm{g} / \mathrm{kg} /$ day) for 1 year. Follow-up: TH profiles, brain MRI and psychometry (Brunet-Lezine) every 3 months. Radioimmunoassay of T4, T3 and TRIAC in cerebrospinal fluid (CSF) of patient and 14 age-matched controls.

Results: Novel de novo MCT8 deletion spanning $25.04 \mathrm{~Kb}$ from mid-exon 3. TRIAC normalized FT3 and TSH $(4.1 \mathrm{pg} / \mathrm{mL}$ and $1.2 \mathrm{mU} / \mathrm{L})$ but reduced FT4 $(0.3 \mathrm{ng} / \mathrm{dL})$. Brain myelination progressed 5 months after 9-months treatment. After initial improvement of motor (18\% to $40 \%)$ and social-language (at 70-72\%) at 3 months, developmental achievements slowed-down at 1 year ( $30 \%$ and $55 \%$, respectively). In CSF, TRIAC was $5 \mathrm{ng} / \mathrm{dL}(\mathrm{N}: 1.4-4.7)$ before trial's start, increasing to $8.4 \mathrm{ng} / \mathrm{dL}$ at highest TRIAC dose. T4 was low (50.2 $\mathrm{ng} / \mathrm{dL}$; N: 65-166), further decreasing under TRIAC (19.2 ng/dL). T3 was low, before and after treatment $(0.7$ and $1.06 \mathrm{ng} / \mathrm{dL} ; \mathrm{N}: 1.9-2.85)$.

Conclusions: TRIAC normalizes serum FT3 and TSH, but decreases FT4, requiring L-T4 substitution. After initial improvement of milestone acquisition, developmental deceleration occurred. Iodothyronines in CSF are low irrespective of treatment, suggesting blood-CSF barrier transport of T3-T4 is restricted in AHDS.

\begin{tabular}{ll}
\hline KARGER & $\begin{array}{l}\text { C } 2014 \text { European Thyroid Association } \\
\text { Published by S. Karger AG, Basel }\end{array}$ \\
& $2235-0640 / 14 / 0035-0249 \$ 39.50 / 0$ \\
E-Mail Karger@karger.com & $\begin{array}{l}\text { Accessible online at: } \\
\text { www.karger.com/etj }\end{array}$
\end{tabular}


WHOLE GENOME SEQUENCE BASED ANALYSIS OF THYROID FUNCTION

Peter N. Taylor ${ }^{1 *}$, Eleonora Porcu, ${ }^{2,3,4 *}$, Shelby Chew ${ }^{5 *}$, Purdey J. Campbel/5*, Michela Traglia 6 , Suzanne J. Brown ${ }^{5}$, Benjamin H. Mullin ${ }^{5,7}$, Hashem A. Shihab ${ }^{8}$, Josine L. Min ${ }^{8}$, Klaudia Walter ${ }^{9}$, Yasin Memari ${ }^{9}$, Jie Huang ${ }^{9}$, Michael R. Barnes ${ }^{10}$, John P. Beilby 11,12, Pimphen Charoen ${ }^{13,14}$, Petr Danecek ${ }^{9}$, Frank Dudbridge ${ }^{13}$, Vincenzo Forgetta ${ }^{15,16}$, Celia Greenwood ${ }^{15,16}$, Elin Grundberg ${ }^{17,18}$, Andrew D. Johnson ${ }^{19}$, Jennie Hui ${ }^{11,12}$, Ee M. Lim ${ }^{5,11}$, Shane McCarthy ${ }^{9}$, Dawn Muddyman ${ }^{9}$, Vijay Panicker ${ }^{5}$, John R.B. Perry ${ }^{20,21}$, Jordana T. BelR1, Wei Yuan ${ }^{21}$, David Schlessinger ${ }^{22}$, Goncalo Abecasis ${ }^{4}$, Francesco Cucca ${ }^{2,3}$, Gabriela L. Surdulescu ${ }^{21}$, Wolfram Woltersdorf ${ }^{23}$, Eleftheria Zeggini ${ }^{9}$, Houfeng Zheng ${ }^{15,16}$, Daniela Toniolo ${ }^{6,24}$, Colin M. Dayan ${ }^{1}$, Silvia Naitza ${ }^{2}$, John P. Walsh ${ }^{5,7}$, Tim D. Spector ${ }^{21}$, George Davey Smith ${ }^{8}$, Richard Durbin ${ }^{9}$, J. Brent Richards ${ }^{15,16,21}$, Serena Sanna ${ }^{2}$, Nicole Soranzo ${ }^{9}$, Nicholas J. Timpson ${ }^{8 *}$, Scott G. Wilson ${ }^{5,7,21 *}$, and the UK10K Consortium *Authors contributed equally

${ }^{1}$ Thyroid Research Group, Institute of Molecular \& Experimental Medicine, Cardiff University School of Medicine, Cardiff University, Cardiff, UK; ${ }^{2}$ Istituto di Ricerca Genetica e Biomedica (IRGB), Consiglio Nazionale delle Ricerche, c/o Cittadella Universitaria di Monserrato, Monserrato, Cagliari, Italy; ${ }^{3}$ Dipartimento di Scienze Biomediche, Università di Sassari, Sassari, Italy; ${ }^{4}$ Center for Statistical Genetics, Biostatistics Department, University of Michigan, Ann Arbor, MI, USA; ${ }^{5}$ Department of Endocrinology and Diabetes, Sir Charles Gairdner Hospital, Nedlands, Western Australia, Australia; ${ }^{6}$ Division of Genetics and Cell Biology, San Raffaele Research Institute, Milano, Italy; ${ }^{7}$ School of Medicine and Pharmacology, University of Western Australia, Crawley, Western Australia, Australia; ${ }^{8} \mathrm{MRC}$ Integrative Epidemiology Unit at the University of Bristol, University of Bristol, Bristol, UK; ${ }^{9}$ Wellcome Trust Sanger Institute, Wellcome Trust Genome Campus, Cambridge, UK; ${ }^{10}$ William Harvey Research Institute, Barts and The London School of Medicine and Dentistry, Queen Mary University of London, London, UK; ${ }^{11}$ Pathwest Laboratory Medicine WA, Nedlands, Western Australia, Australia; ${ }^{12}$ School of Pathology and Laboratory Medicine, University of Western Australia, Crawley, Western Australia, Australia; ${ }^{13}$ Faculty of Epidemiology and Population Health, London School of Hygiene and Tropical Medicine, London, UK; ${ }^{14}$ Department of Tropical Hygiene, Faculty of Tropical Medicine, Mahidol University, Bangkok, Thailand; ${ }^{15}$ Department of Medicine, Jewish General Hospital, McGill University, Montréal Québec, Canada; ${ }^{16}$ Departments of Human Genetics, Epidemiology, and Biostatistics, Jewish General Hospital, Lady Davis Institute, McGill University, Montréal Québec, Canada; ${ }^{17}$ Department of Human Genetics, McGill University, Montreal, QC, Canada; ${ }^{18}$ McGill University and Genome Quebec Innovation Centre, Montreal, QC, Canada; ${ }^{19} \mathrm{Cardiovascular}$ Epidemiology and Human Genomics Branch, National Heart, Lung and Blood Institute, Bethesda, MD, USA; ${ }^{20}$ MRC Epidemiology Unit, University of Cambridge School of Clinical Medicine, Institute of Metabolic Science, Cambridge Biomedical Campus, Cambridge, UK; ${ }^{21}$ Department of Twin Research and Genetic Epidemiology, King's College London, London, UK; ${ }^{22}$ Laboratory of Genetics, NIA, Baltimore, MD, USA; ${ }^{23}$ Facharzt für Laboratoriumsmedizin, Geschäftsführer amedes Ost, Halle/Leipzig GmbH, Halle (Saale), Germany; ${ }^{24}$ Institute of Molecular Genetics-CNR, Pavia, Italy

Objective: The genetic architecture of thyroid function is poorly understood. We aimed to identify genetic variants, including rare variants, associated with thyrotropin (TSH) and free thyroxine (FT4) using whole genome sequencing (WGS) data from the UK10K Consortium.

Methods: TSH and FT4 levels were standardized adjusting for age, age ${ }^{2}$ and sex. We analyzed data from two UK10K cohorts (ALSPAC and TwinsUK
$\mathrm{N}=2,287$ ) and used an additional collection with WGS data (SardiNIA) and deeply imputed datasets (imputed to a joint 1000 genomes and UK10K reference panel) to perform a meta-analysis for common variants (MAF $>1 \%$ ) associated with TSH and FT4 $(\mathrm{N}=16,335)$. We undertook analysis of exonic rare variants ( $\mathrm{MAF}<1 \%$ ) using sequence kernel association testing (SKAT) in 40 candidate genes and performed genome-wide complex trait analyses (GCTA) to explore the extent that common SNPs (MAF $>1 \%$ ) explained the variance in TSH and FT4.

Results: For TSH we report a novel variant at $3 \mathrm{p} 25(\mathrm{MAF}=23.5 \%, \mathrm{P}=$ $\left.6.15 \times 10^{-9}\right)$ and a new independent variant in PDE8B $(\mathrm{MAF}=10.4 \%, \mathrm{P}=$ $\left.5.94 \times 10^{-14}\right)$. Expression quantitative trait locus analysis revealed our variant at 3 p25 modulates gene transcription in adipose, skin and whole blood cells. Methylation profiles revealed evidence for methylation quantitative trait locus effects for our novel variant in $P D E 8 B\left(\mathrm{P}=4.38 \times 10^{-7}\right)$. For FT4 we identified a low frequency variant in $18 \mathrm{q} 11\left(\mathrm{MAF}=3.2 \%, \mathrm{P}=1.27 \times 10^{-9}\right)$ tagging a rare functional variant in TTR $\left(\mathrm{MAF}=0.4 \%, \mathrm{P}=2.14 \times 10^{-11}\right)$. SKAT analysis revealed a novel association with FT4 in chromosome $8 \mathrm{p} 12\left(\mathrm{P}=2.53 \times 10^{-6}\right)$. GCTA analysis estimated common SNPs (MAF $>1 \%$ ) explained $24 \%(95 \%$ CI 19,29$)$ and $20 \%(95 \%$ CI 14,26$)$ of TSH and FT4 variance, respectively $(\mathrm{P} \leq 0.0001)$.

Conclusion: Our results demonstrate that increased coverage in WGS population association studies allows detection of both common and rare variants in thyroid function. Common variants collectively account for over $20 \%$ of the variance in TSH and FT4; a substantial advance on estimates from earlier genome-wide association studies.

\section{EFFECTS OF EARLY LT4 TREATMENT IN A PATIENT WITH A MUTATION IN TR $\alpha 1 / \alpha 2$}

Anja L.M. van Gucht ${ }^{1}$, Nitash Zwaveling-Soonawala²,

Marcel E. Meima', W. Edward Visser', Theo J. Visser',

Robin P. Peeters ${ }^{1}$, A.S. Paul van Trotsenburg ${ }^{2}$

${ }^{1}$ Department of Internal Medicine, Erasmus University Medical Center, Rotterdam, The Netherlands; ${ }^{2}$ Department of Pediatric Endocrinology, Emma Children's Hospital, Academic Medical Center, Amsterdam, The Netherlands

Background: Patients with resistance to thyroid hormone due to inactivating mutations in $\mathrm{TR} \alpha 1(\mathrm{RTH} \alpha)$ are characterized by growth retardation, delayed bone and motor development, cognitive defects, and abnormal TFTs. Recently, the first patients with a mutation in both TR $\alpha 1$ and its non-T3-binding splice variant TR $\alpha 2$ have been identified. Here, we describe a young girl (15 mo) with a mutation in both TR $\alpha 1$ and TR $\alpha 2$.

Objective: To study the effects of early LT4 treatment.

Method: The patient was assessed before and during 6 mo of LT4 treatment, started at the age of 18 mo. In addition, we studied transcriptional activity of mutant (MT) vs. wild-type (WT) TR $\alpha 1$ in cells co-transfected with a TR-dependent promoter-reporter construct.

Results: At $15 \mathrm{mo}$, the patient presented with axial hypotonia, delayed motor development (Alberta Infant Motor Scale (AIMS) $<$ P5) and severe growth retardation (height $-2.6 \mathrm{SDS}$ ). She also showed low serum (F)T4 and rT3, increased T3 and normal TSH levels. The patient has a D211G mutation in both TR $\alpha 1$ and TR $\alpha 2$, resulting in decreased transcriptional activity of TR $\alpha 1$, overcome at higher T3 levels (MT vs. WT TR $\alpha 1$ activity was $46 \%$ at $1 \mathrm{nM} \mathrm{T} 3$, and $78 \%$ at $100 \mathrm{nM} \mathrm{T3}$ ). MT TR 1 showed little if any dominant activity towards WT TR $\alpha 1$. In addition, WT or MT TR $\alpha 2$ did not show a significant dominant-negative inhibition of WT or MT TR $\alpha 1$.

Six mo of LT4 treatment $(3.5-4.7 \mu \mathrm{g} / \mathrm{kg})$ resulted in a marked improvement of the hypotonia, motor development (AIMS normalized) and growth (height increase of $0.76 \mathrm{SDS}$ ). In addition, serum TSH was suppressed, (F)T4 and rT3 normalized, and T3 increased slightly.

Conclusion: This case emphasizes the importance of identifying patients with TR $\alpha$ mutations at a very young age, since early LT4 treatment may lead to clear clinical improvement. 